\title{
A NEW CHARACTERIZATION OF REFLEXIVITY
}

\section{RUIDONG WANG}

\author{
(Received 25 February 2008)
}

\begin{abstract}
In this paper, we give a new characterization of reflexive Banach spaces in terms of the sum of two closed bounded convex sets.
\end{abstract}

2000 Mathematics subject classification: primary 46B20.

Keywords and phrases: reflexive, bounded convex closed set.

We know that the sum of a compact set and a closed set is closed; it is also known that the sum of two closed sets need not to be closed. In this paper we shall show that reflexivity of a Banach space can be characterized by the property that the sum of any two closed bounded convex sets in the Banach space remains closed.

Throughout this paper, $E$ will be a Banach space, and we shall use $S(E)$ and $B(E)$ to denote the unit sphere of $E$ and the unit ball of $E$, respectively. Now we present our main theorem.

MAIN THEOREM. The Banach space $E$ is reflexive if and only if the sum of any two closed bounded convex sets in E is still closed.

ProOf. First, assume that $E$ is reflexive. Let $A, B \subset E$ and suppose that both of these sets are closed bounded convex sets. Then $A$ and $B$ are compact in the weak topology of $E$, and hence $A+B$ is closed in the weak topology of $E$. It is obvious that $A+B$ is convex, so we deduce that $A+B$ is closed in the norm topology.

To prove the converse, suppose that $E$ is not reflexive. Then, by James's well-known characterization of reflexivity in terms of the supremum of linear functionals [1], there exists $x^{*} \in S\left(E^{*}\right)$ such that $x^{*}$ does not attain its norm on $B(E)$. Let $\left\{x_{n}\right\} \subset B(E)$ such that

$$
x^{*}\left(x_{n}\right)>1-\frac{1}{2^{n}} \text { for all } n \in \mathbb{N} .
$$

The author was supported by the NSFC (grant no. 10571090) and the Doctoral Programme Foundation of the Institution of Higher Education (grant no. 20060055010).

(C) 2009 Australian Mathematical Society 0004-9727/09 \$A2.00+0.00 
Set $H=\left\{x \in E: x^{*}(x)=1\right\}$. It is easy to see that $H \cap B(E)=\emptyset$.

Now fix $x_{0} \in H$. For $n \in \mathbb{N}$, let $y_{n}=x_{n}+\left(1-x^{*}\left(x_{n}\right)\right) x_{0}$; then $x^{*}\left(y_{n}\right)=1$ and

$$
\left\|x_{n}-y_{n}\right\|=\left\|\left(1-x^{*}\left(x_{n}\right)\right) x_{0}\right\|<\frac{1}{2^{n}}\left\|x_{0}\right\| .
$$

Letting $B=\overline{c o}\left\{-x_{n}\right\}$ and $A=\overline{c o}\left\{y_{n}\right\}$, we have $A \subset H$ and $-B \subset B(E)$.

Since

$$
\lim _{n \rightarrow \infty}\left\|x_{n}-y_{n}\right\|=0
$$

we obtain $\theta \in \overline{A+B}$.

But $A \cap(-B)=\emptyset$, so it must be that $\theta \notin A+B$.

Therefore $A+B$ is not closed, which contradicts our assumption. Thus we conclude that $E$ is reflexive.

\section{References}

[1] R. C. James, 'Reflexivity and the sup of linear functionals', Israel J. Math. 13 (1972), 289-300.

RUIDONG WANG, School of Mathematics, Nankai University, Tianjing 300071, People's Republic of China

e-mail: wangruidong@mail.nankai.edu.cn 